\title{
Mechanisms of Charge Accumulation in the Dark Operation of Perovskite Solar Cells
}

\author{
Teresa S. Ripolles, ${ }^{* 1}$ Ajay K. Baranwal, ${ }^{1}$ Koji Nishinaka, ${ }^{1}$ Yuhei Ogomi, ${ }^{1}$ Germà Garcia- \\ Belmonte, ${ }^{2}$ and Shuzi Hayase ${ }^{* 1}$ \\ ${ }^{1}$ Graduate School of Life Science and Systems Engineering, Kyushu Institute of Technology, \\ 2-4 Hibikino, Wakamatsu-ku, Kitakyushu, Fukuoka 808-0196, Japan. \\ ${ }^{2}$ Institute of Advance Materials (INAM), Universitat Jaume I, ES-12006 Castelló, Spain.
}

\begin{abstract}
In this work, a new current peak at forward bias in the dark current-voltage curves has been identified for standard mesoscopic perovskite solar cells. This characteristic peak appears only under some specific conditions, mainly at reverse scan (RS) direction and when the solar cells were kept for several seconds at short-circuit conditions before starting the RS measurement. This peak disappears when the above experimental conditions are not applied. It is considered that this uncommon diode shape occurs because shallow and/or deep trap states located at the interface between either perovskite/p-type or perovskite/n-type transport materials are dynamically filled during RS voltage scan. To corroborate this hypothesis, the response of hole transport materials (HTMs) small molecule spiro-OMeTAD and polymer P3HT, as well as both HTMs with additives, were compared. Also perovskite absorbers as $\mathrm{CH}_{3} \mathrm{NH}_{3} \mathrm{PbI}_{3}\left(\mathrm{MAPbI}_{3}\right)$ and all-inorganic perovskite based on cesium $\left(\mathrm{CsPbI}_{3}\right)$ were also analyzed, achieving in all cases similar trends.
\end{abstract}

Keywords: Perovskite, traps, charge accumulation, dark.

*Corresponding authors:

T. S. Ripolles, e-mail address: teresa@ life.kyutech.ac.jp

G. Garcia-Belmonte, e-mail address: garciag@uji.es

S. Hayase, e-mail address: hayase@life.kyutech.ac.jp

4 February 2016 
The rapid development of organometal halide perovskite solar cells since six years ago has yielded an attractive technology able to be an alternative to conventional photovoltaic solar cells. ${ }^{1}$ Solid state solar cells with high efficiencies over $21 \%$, numerous new materials and interfaces, easy fabrication, and alternative device configurations are the reasons of their major progress so far. ${ }^{2-3}$ However, there are still some issues that should be addressed to well understand the working mechanisms of these devices. The electrical processes such as carrier transport into the perovskite film and charge extraction at the interfaces as well an electric field that occur on the timescale of seconds are still under debate. ${ }^{4}$ Fundamental device physics issues are of great interest because they determine the photoconversion efficiency. Particularly, several reports were focused on the trapping of electronic carriers most likely at the interface between perovskite and $n$-type extraction material. ${ }^{5}$ This factor is highly dependent on the device architecture. As regards mesoporous $\mathrm{CH}_{3} \mathrm{NH}_{3} \mathrm{PbI}_{3}$-based perovskite solar cells, the surface trap density can be reduced when the surface of the $\mathrm{TiO}_{2}$ nanoparticles was passivated with lithium. ${ }^{6}$ Another option to suppress the intrinsic traps sites was introduced as core/shell-structured $\mathrm{CdS} / \mathrm{TiO}_{2}$, reducing the decomposition of the perovskite absorber that occurs under illumination. ${ }^{7}$ On the other hand, to facilitate the charge transfer at the interface from perovskite to $\mathrm{TiO}_{2}$, and suppress the accumulation charge at the perovskite, planar architecture can be an alternative when PCBM was added between compact- $\mathrm{TiO}_{2}\left(\mathrm{c}-\mathrm{TiO}_{2}\right)$ and the perovskite film. Concerning trap states in the $\mathrm{c}-\mathrm{TiO}_{2},{ }^{8}$ the $\mathrm{PCBM}$ plays a passivation role at iodide-rich trap sites on the surface of the perovskite grains. ${ }^{9}$ Recent analysis suggests accumulation of carriers at interfaces at forward bias under illumination. ${ }^{10}$ Similarly, high positive voltage might induce large carrier injection and consequently charge accumulation even for dark conditions. The above cases were focused on the electron extraction interface. However, this study is addressed to the chemical interactions and physical mechanisms occurring at interlayer between perovskite and hole transport material which is less investigated till now.

In this work, a series of electrical experiments using solar cells with a configuration as glass/FTO/c- $-\mathrm{TiO}_{2} / \mathrm{mp}-\mathrm{TiO}_{2} /$ perovskite/HTM/Au, where FTO is fluorine doped tin oxide, $\mathrm{c}-\mathrm{TiO}_{2}$ is the compact layer of $\mathrm{TiO}_{2}, \mathrm{mp}-\mathrm{TiO}_{2}$ is the mesoporous layer of $\mathrm{TiO}_{2}$, and HTM is the hole transport material, were carried out in order to understand distinctive current-voltage features and underlying mechanisms. The absorber layers analyzed in this study were the standard methyl ammonium lead iodine, $\mathrm{CH}_{3} \mathrm{NH}_{3} \mathrm{PbI}_{3}\left(\mathrm{MAPbI}_{3}\right)$, with an intrinsic dipole from the $\mathrm{MA}^{+}$and the all-inorganic cesium lead iodine $\left(\mathrm{CsPb}_{3}\right)$. Additionally, several interlayer hole-extracting materials were checked: either the standard small molecule 2,2',7,7'-tetrakis $\left(N, N^{\prime}\right.$-di- $p$ methoxyphenylamine)-9,9'-spirobifluorene (spiro-OMeTAD) or the polymer poly(3hexylthiophene) (P3HT), allowing for a variation of the interface work function. Every photovoltaic device was characterized by current-voltage $(I-V)$ curves under dark at reverse voltage sweep for several voltage-time preconditioning. Our findings suggest the trapping or accumulation of charge carriers at the interlayers as a general mechanism observed for different perovskite absorbers and interlayer materials. 
Figure 1a shows the $I-V$ characteristics under $100 \mathrm{mWcm}^{-2}$ air mass 1.5 global (AM $1.5 \mathrm{G}$ ) for the standard perovskite solar cell with the architecture such as glass/FTO/c- $\mathrm{TiO}_{2} / \mathrm{mp}$ $\mathrm{TiO}_{2} / \mathrm{MAPbI}_{3} /$ spiro-OMeTAD/Au. All experimental details are specified in the Supporting Information. This measurement was performed in a forward sweep (FS) from negative applied bias $\left(-V_{\text {app }}\right)$ to positive applied bias $\left(+V_{\text {app }}\right)$ and reverse sweep (RS) from $+V_{\text {app }}$ to $-V_{\text {app. All }}$ photovoltaic results are summarized in Table 1 . An overestimation of power conversion efficiencies (PCEs) of $14.18 \%$ in FS to $14.87 \%$ in RS was achieved due to the inherent hysteresis effect.

However, the current-voltage curves scanned at FS and RS under dark conditions are still less studied. In particular, from short-circuit conditions to large forward voltages $(+3.5 \mathrm{~V})$ and back, keeping the system between these two measurements at short-circuit conditions or waiting for several seconds at forward bias. Figure 1b shows the current-voltage performance in the dark at forward and backward sweep directions measured at different experimental conditions, such as applying a delay time between FS and RS measurements for $0 \mathrm{~s}$ and $20 \mathrm{~s}$ at $+3.5 \mathrm{~V}$ and $20 \mathrm{~s}$ at 0 $\mathrm{V}$. The $j-V$ curve shows the expected diode shape when the reverse scan was performed immediately after finishing forward scan (delay time $0 \mathrm{~s}$ ). Conversely, a new shape appears when the solar cell was short-circuited for $20 \mathrm{~s}$ prior to starting the measurement from forward bias at $+3.5 \mathrm{~V}$ to short-circuit (Figure $1 \mathrm{~b}$ ). A distinctive current peak is found at voltages slightly below $+3.5 \mathrm{~V}$ which produces a reduction in the measured current with respect to that measured in the FS. This effect is enhanced by increasing the waiting time between FS-RS measurements (Figure 1c). As next explained this behavior indicates the presence of traps able to store charge carriers either at perovskite absorber film, ${ }^{11}$ at the boundary of the $\mathrm{TiO}_{2}$ nanoparticles with the absorber ${ }^{6-}$ ${ }^{7}$ or at the interface between the perovskite and HTL. Based on these results, it is proposed that shadow traps located at charge extraction interface either $\mathrm{TiO}_{2}$ nanoparticles or hole transport layer are being filled at the forward scan. Consequently, when the reverse scan was measured immediately after finishing the forward scan, or even keeping the solar cell several seconds at forward bias (Figure $1 \mathrm{~b}$ for $20 \mathrm{~s}$ and Figure SI 1 for several seconds), the usual diode shape of the $j$ - $V$ curve occurs because all traps are filled since the beginning of the RS measurement. However, as waiting time was increased from $5 \mathrm{~s}$ to $60 \mathrm{~s}$ at short-circuit conditions, the intensity of the current peak is enlarged because more traps have to be filled once again to recover the current level at forward bias.

The previous results are highly affected by the kinetics of the photovoltaic performance because the scan rate determines different state of the solar cell. ${ }^{12}$ Figure 2 represents the $j$ - $V$ curves under dark at reverse for two different voltage scan rates at $0.1 \mathrm{~V} / \mathrm{s}$ and at $0.01 \mathrm{~V} / \mathrm{s}$. The preconditions of the reverse measurement were maintained in holding the device for several seconds $(20 \mathrm{~s}$ or $60 \mathrm{~s}$ ) at short-circuit conditions or at forward bias (denoted as $0 \mathrm{~s}$ ). Higher intensity of current peaks is observed because the device takes longer to reach the quasi-steadystate. Therefore, more charge carriers are accumulated at slow scan rate when the delay time 
between forward and reverse measurements was several seconds, compared with the fast scan rate measurements. This implies that current level recovering is favored by slow scans.

In order to further check the mechanism behind the observed $j-V$ curve distortion for this kind of solar cells, other mp- $\mathrm{TiO}_{2}$ device structures will be analyzed. Particularly, different materials at anode interface were studied, such as the polymer P3HT instead of the small molecule spiroOMeTAD, with both HTMs being altered with some chemicals as additives (more details in the Supporting Information). Additionally, another factor that will be considered is the perovskite absorber material, being $\mathrm{MAPbI}_{3}$ contrasted with all-inorganic cesium perovskite as $\mathrm{CsPbI}_{3}$.

The $j$ - $V$ curves under dark in the backward direction by keeping the solar cells at short-circuit conditions for $1 \mathrm{~min}$ before starting the reverse measurement for standard $\mathrm{MAPbI}_{3}$ perovskite compound show different behaviors depending on the hole conductors (Figure 3a). The solar cells with spiro-OMeTAD exhibit high current intensity peaks compared with their counterpart $\mathrm{P} 3 \mathrm{HT}$. The uncommon diode shape is independent of the high applied voltage limit as is represented in Figure 3b.

The current offset at $+3.5 \mathrm{~V}$ achieved at reverse direction respect to the forward direction (see Figure $2 \mathrm{~b}$ ) is related to the reduction in charge accumulation at the interfaces. We propose that the relative percentage of charge accumulation ( $(\mathrm{FS}$ at $+3.5 \mathrm{~V})-\mathrm{I}(\mathrm{RS}$ at $+3.5 \mathrm{~V})) / \mathrm{I}(\mathrm{FS}$ at $+3.5 \mathrm{~V}) \times 100$ ) between the forward and reverse measurements were calculated for the spiroOMeTAD and their derivatives $\mathrm{MAPbI}_{3}$ solar cells. A reduction of the charge accumulation $(14.8 \%)$ for the spiro-OMeTAD respect to the spiro-OMeTAD with additives $(18.0 \%)$ entails that charge carriers are easily removed from the interface between $\mathrm{MAPbI}_{3} / \mathrm{HTM}$ without additives. However, the effect of the co-addition Li-TFSI(TBP) and organic dye based on Co(III) (FK209) as p-dopant into the spiro-OMeTAD solution has reported an improvement in the charge transport properties compared to pristine spiro-OMeTAD. ${ }^{13}$ Additionally, the appointed device was also analyzed at different scan rates and delay times at short-circuit conditions prior starting the RS measurement. This data was summarized in the Supporting Information (see Figure SI 2). Slow $(0.01 \mathrm{~V} / \mathrm{s})$ and fast $(0.1 \mathrm{~V} / \mathrm{s})$ scan rates show two distinct behaviors, mainly low current intensities, as well as the slope of the measurements scanned at low voltage scan rate. However, the opposite behavior is showed for the cells based on spiro-OMeTAD doped solely with Li-TFSI(TBP) (Figure 2). Therefore, different mechanisms are observed at the interface between perovskite $\mathrm{MAPbI}_{3}$ and $\mathrm{HTM}$, being crucial the chemicals used in order to extract and transport charge efficiently.

In addition, similar studies were carried out for $\mathrm{MAPbI}_{3}$ perovskite solar cells with $\mathrm{P} 3 \mathrm{HT}$ as HTM. Figure SI 3 and Figure SI 4 represent the $j$ - $V$ curves under dark at the reverse direction for devices with HTMs of pure P3HT and P3HT with Li-TFSI as an additive, respectively. Unlike the cases described above, similar current intensities are observed, which means that the addition of lithium does not alter significantly the interlayer between perovskite and HTM. 
Once the interlayer between $\mathrm{MAPbI}_{3}$ and HTM was analyzed, the perovskite absorber film is another important factor to take into account. Figure 3a shows the significant current intensity difference between $\mathrm{MAPbI}_{3}-$ and $\mathrm{CsPbI}_{3}$-based solar cells. The fabrication of all-inorganic cesium solar cells was described in our previous publications. ${ }^{14}$ In order to analyze the effect of the $\mathrm{MA}^{+}$and $\mathrm{Cs}^{+}$cations, the reduction in charge accumulation percentage were measured as explained above, being $10.9 \%$ and $18.6 \%$ for the $\mathrm{MAPbI}_{3} / \mathrm{P} 3 \mathrm{HT}$ and $\mathrm{CsPb}_{3} / \mathrm{P} 3 \mathrm{HT}$ solar cells, respectively. Additionally, this effect was increased (37.6\%) when a new layer of molybdenum trioxide $\mathrm{MoO}_{3}$ was deposited between $\mathrm{P} 3 \mathrm{HT}$ and $\mathrm{Au}$ in the $\mathrm{CsPbI}_{3}$ devices.

Summarizing all previous data, the measurements of the current-voltage curves were strongly influenced by several parameters, mainly, (i) the initial state of the solar cell before starting a scan, such as in the dark, and at short-circuit or at forward bias conditions for several seconds, (ii) the voltage sweep rate that in this study was analyzed $0.1 \mathrm{~V} / \mathrm{s}$ and $0.01 \mathrm{~V} / \mathrm{s}$, but commonly was in the range from $>0.1 \mathrm{~V} / \mathrm{s}$ to $0.001 \mathrm{~V} / \mathrm{s},{ }^{12}$ and (iii) the scan direction from short-circuit conditions to forward bias (denoted as forward scan) or the opposite way (denoted as reverse or backward scan).

The phenomenon previously introduced is rationalized in Figure 4, where some sketches are drawn in order to clarify the fundamental mechanisms for the devices that have been studied here. Initially, the current-voltage curves were measured at forward scan direction, followed by either keeping the solar cell at forward bias for several seconds and then measure the $I-V$ curve at reverse scan direction, or measure the $I-V$ curve at RS directly (Figure $4 \mathrm{a}$ ). In both cases, the initial experimental conditions for the solar cell are the same, namely forward bias. The $I-V$ curves (Figure 4b) showed under the experimental conditions explained above present the characteristic diode shape at forward and reverse bias directions. We consider that at forward sweep direction (Figure 4c), the shallow or deep traps located at the interface between perovskite absorber and hole transport layers as well as at the boundary of the $\mathrm{TiO}_{2}$ nanoparticles, are filled with applied voltage. Consequently, taking into account the preconditions before starting the RS scan, the $I-V$ curve at reverse direction started without empty traps (Figure 4d). On the contrary, a new shape of the $I-V$ curve was observed at RS (Figure $4 \mathrm{f}$ ) when the solar cell was kept at short-circuit conditions for several seconds between forward and reverse $I$ - $V$ curve measurements under dark (Figure 4e). At short-circuit conditions, shallow or deep traps have time to gradually empty out depending on the delay time as represented in Figure $4 \mathrm{~g}$. Once the reverse scan starts, the traps should be filled once again (Figure 4h), showing the noncharacteristics diode shape. It should be stressed here that our experiments are not conclusive with respect to which outer contact (either perovskite/TiO 2 or perovskite/HTM) are responsible for the charge carrier trapping. Changing the HTM or the perovskite material induces large variations in the dark current level in the forward sweep, the position, and height of the reverse sweep peak is then modified. In addition, other explanations different from the charge trapping discussed here are also plausible as the recently proposed occurrence of carrier accumulation zones at the interfaces. ${ }^{10}$ 
In summary, we report that the experimental preconditions of the current-voltage curves under dark play a crucial role in order to understand the physical mechanisms that occur in the $\mathrm{MAPbI}_{3} /$ spiro-OMeTAD-based perovskite solar cells. The scan rate, the bias sweep direction, as well as, preconditioning the solar cell were the main parameters analyzed in this manuscript. In particular, a new peak at forward bias in the $I-V$ curve was shown at reverse sweep bias direction after the system was equilibrated at short-circuit conditions for several seconds. The current intensity peak was controlled depending on the delay time under short-circuit conditions and the scan rate. This uncommon diode shape was attributed to the shallow and/or deep traps located at interface perovskite/ $p$ - and/or $n$-type contact materials. In order to corroborate those results, other materials with different work functions were analyzed, achieving similar trends.

\section{ASSOCIATED CONTENT}

Supporting Information. Details of experiments and additional supplementary figures. This material is available free of charge via the Internet.

\section{Notes}

The authors declare no competing financial interest.

\section{ACKNOWLEDGMENT}

This research was supported by CREST. 


\section{References}

1. Kojima, A.; Teshima, K.; Shirai, Y.; Miyasaka, T., Organometal Halide Perovskites as Visible-Light Sensitizers for Photovoltaic Cells. Journal of the American Chemical Society 2009, 131 (17), 6050-+.

2. Jung, H. S.; Park, N. G., Perovskite Solar Cells: From Materials to Devices. Small 2015, $11(1), 10-25$.

3. Park, N. G., Perovskite solar cells: an emerging photovoltaic technology. Mater. Today 2015, $18(2), 65-72$.

4. Shi, J. J.; Xu, X.; Zhang, H. Y.; Luo, Y. H.; Li, D. M.; Meng, Q. B., Intrinsic slow charge response in the perovskite solar cells: Electron and ion transport. Appl. Phys. Lett. 2015, 107 (16), 5 .

5. Snaith, H. J.; Abate, A.; Ball, J. M.; Eperon, G. E.; Leijtens, T.; Noel, N. K.; Stranks, S. D.; Wang, J. T. W.; Wojciechowski, K.; Zhang, W., Anomalous Hysteresis in Perovskite Solar Cells. J. Phys. Chem. Lett. 2014, 5 (9), 1511-1515.

6. Heo, J. H.; You, M. S.; Chang, M. H.; Yin, W.; Ahn, T. K.; Lee, S. J.; Sung, S. J.; Kim, D. H.; Im, S. H., Hysteresis-less mesoscopic $\mathrm{CH}(3) \mathrm{NH}(3) \mathrm{Pbl}(3)$ perovskite hybrid solar cells by introduction of Li-treated TiO2 electrode. Nano Energy 2015, 15, 530-539.

7. Hwang, I.; Baek, M.; Yong, K., Core/Shell Structured TiO2/CdS Electrode to Enhance the Light Stability of Perovskite Solar Cells. ACS Applied Materials \& Interfaces 2015, 7 (50), 27863-27870.

8. Jena, A. K.; Chen, H. W.; Kogo, A.; Sanehira, Y.; Ikegami, M.; Miyasaka, T., The Interface between FTO and the TiO2 Compact Layer Can Be One of the Origins to Hysteresis in Planar Heterojunction Perovskite Solar Cells. ACS Applied Materials \& Interfaces 2015, 7 (18), 9817-9823.

9. Xu, J.; Buin, A.; Ip, A. H.; Li, W.; Voznyy, O.; Comin, R.; Yuan, M.; Jeon, S.; Ning, Z.; McDowell, J. J.; Kanjanaboos, P.; Sun, J.-P.; Lan, X.; Quan, L. N.; Kim, D. H.; Hill, I. G.; Maksymovych, P.; Sargent, E. H., Perovskite-fullerene hybrid materials suppress hysteresis in planar diodes. Nature Communications 2015, 6.

10. Zarazua, I.; Bisquert, J.; Garcia-Belmonte, G., Light-Induced Space-Charge Accumulation Zone as Photovoltaic Mechanism in Perovskite Solar Cells. J. Phys. Chem. Lett. 2016, 7, 525-528.

11. Wu, B.; Fu, K. W.; Yantara, N.; Xing, G. C.; Sun, S. Y.; Sum, T. C.; Mathews, N., Charge Accumulation and Hysteresis in Perovskite-Based Solar Cells: An Electro-Optical Analysis. Adv. Energy Mater. 2015, 5 (19), 8. 
12. Tress, W.; Marinova, N.; Moehl, T.; Zakeeruddin, S. M.; Nazeeruddin, M. K.; Gratzel, M., Understanding the rate-dependent J-V hysteresis, slow time component, and aging in $\mathrm{CH} 3 \mathrm{NH} 3 \mathrm{PbI} 3$ perovskite solar cells: the role of a compensated electric field. Energy Environ. Sci. 2015, 8 (3), 995-1004.

13. Noh, J. H.; Jeon, N. J.; Choi, Y. C.; Nazeeruddin, M. K.; Gratzel, M.; Seok, S. I., Nanostructured TiO2/CH3NH3PbI3 heterojunction solar cells employing spiro-OMeTAD/Cocomplex as hole-transporting material. Journal of Materials Chemistry A 2013, 1 (38), 1184211847.

14. Ripolles, T. S.; Nishinaka, K.; Ogomi, Y.; Miyata, Y.; Hayase, S., Efficiency enhancement by changing perovskite crystal phase and adding a charge extraction interlayer in organic amine free-perovskite solar cells based on cesium. Solar Energy Materials \& Solar Cells 2016, 144, 532-536. 
Table 1. Photovoltaic parameters under simulated 1 sun illumination at forward and reverse directions for the standard device glass/FTO/c-TiO $2 / \mathrm{mp}-\mathrm{TiO}_{2} / \mathrm{MAPbI}_{3} /$ spiro-OMeTAD/Au.

\begin{tabular}{ccccc}
\hline Sweep & $\mathrm{j}_{\mathrm{sc}}, \mathrm{mAcm}^{-2}$ & $\mathrm{~V}_{\mathrm{oc}}, \mathrm{V}$ & $\mathrm{FF}$ & PCE, \% \\
\hline Forward & 21.91 & 1.06 & 0.61 & 14.18 \\
Reverse & 20.79 & 1.05 & 0.68 & 14.87 \\
\hline
\end{tabular}




\section{List of Figures}

Figure 1. Current density-voltage curves (a) at simulated 1 sun illumination from short-circuit to forward conditions (forward sweep, FS) and forward to short-circuit conditions (reverse sweep, $\mathrm{RS}$ ); (b) under dark conditions at FS and RS, the solar cell remains at $+3.5 \mathrm{~V}$ or short-circuit conditions for $0 \mathrm{~s}$ or $20 \mathrm{~s}$ between the measurements FS and RS; and, (c) under dark at RS and at different delay times between FS and RS measurements. The scan rate was $0.1 \mathrm{~V} / \mathrm{s}$ for all measurements.

Figure 2. Current density-voltage curves under dark at different scan rates such as $0.1 \mathrm{~V} / \mathrm{s}$ (solid line) and $0.01 \mathrm{~V} / \mathrm{s}$ (dashed line). Every scan starts from forward bias to short-circuit and prior to scanning, the device was stabilized some seconds $(0,20$ or $60 \mathrm{~s})$ at short-circuit conditions. The zoom of (a) is showed in (b).

Figure 3. (a) The $j-V$ curves for perovskite solar cells with different absorber material and HTMs under dark at the reverse direction, the scan rate was $0.1 \mathrm{~V} / \mathrm{s}$ and the delay time was 1 min at shortcircuit conditions prior RS measurements. (b) The $j$ - $V$ curves under dark at RS with different starting bias and delay time was $10 \mathrm{~s}$ for the device based on $\mathrm{MAPbI}_{3} / \mathrm{P} 3 \mathrm{HT}$ solar cell.

Figure 4. Schematic representation of the conditions of the $I-V$ measurements (a) and (e), $I-V$ curves (b) and (f), and energy level diagrams (c), (d), (g), and (h). The above and below images represent the experiment without and with a delay time between forward and reverse bias direction in the $I$ - $V$ curves, respectively. These mechanisms represent only the electron trapping at the interface of perovskite/HTM. 
1
2

(a)

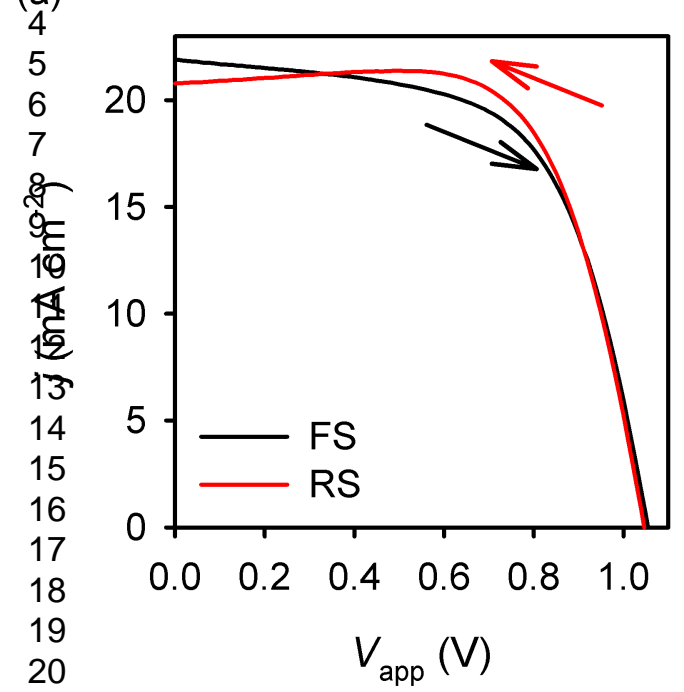

(b)

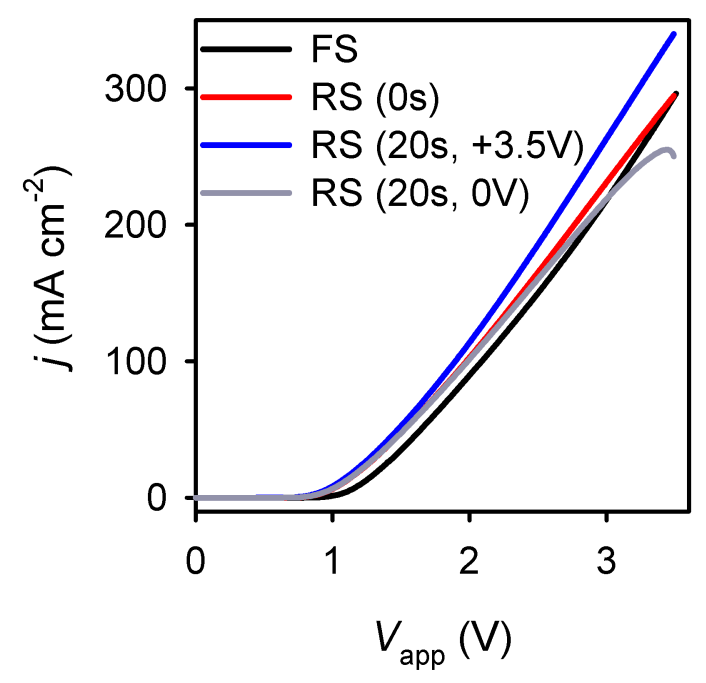

Figure 1 (c)

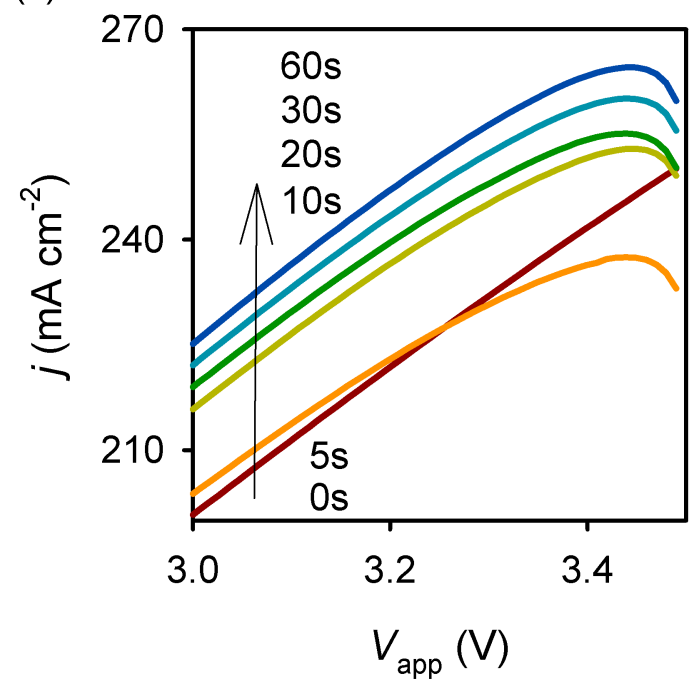


(a)

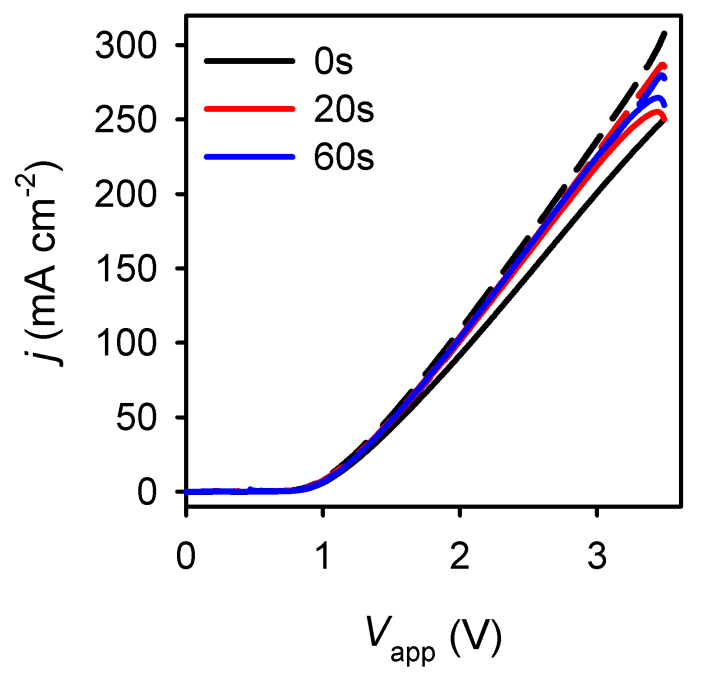

(b)

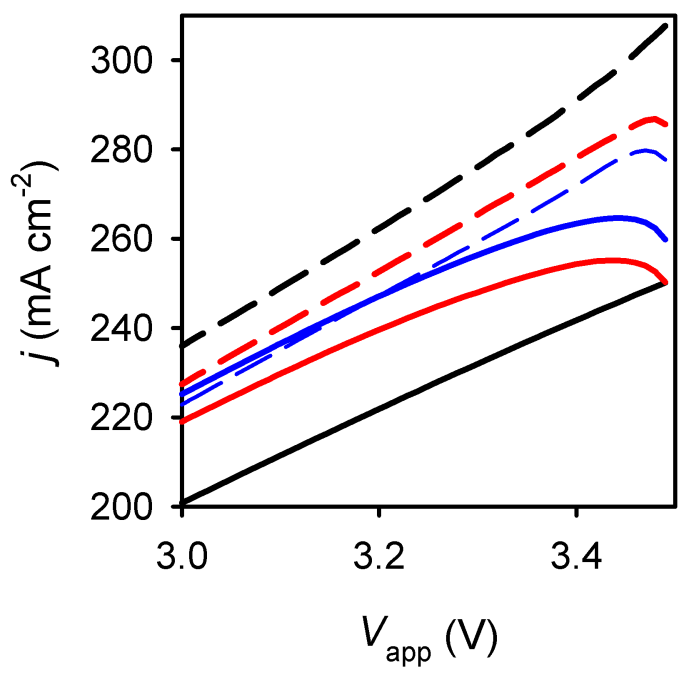

Figure 2 

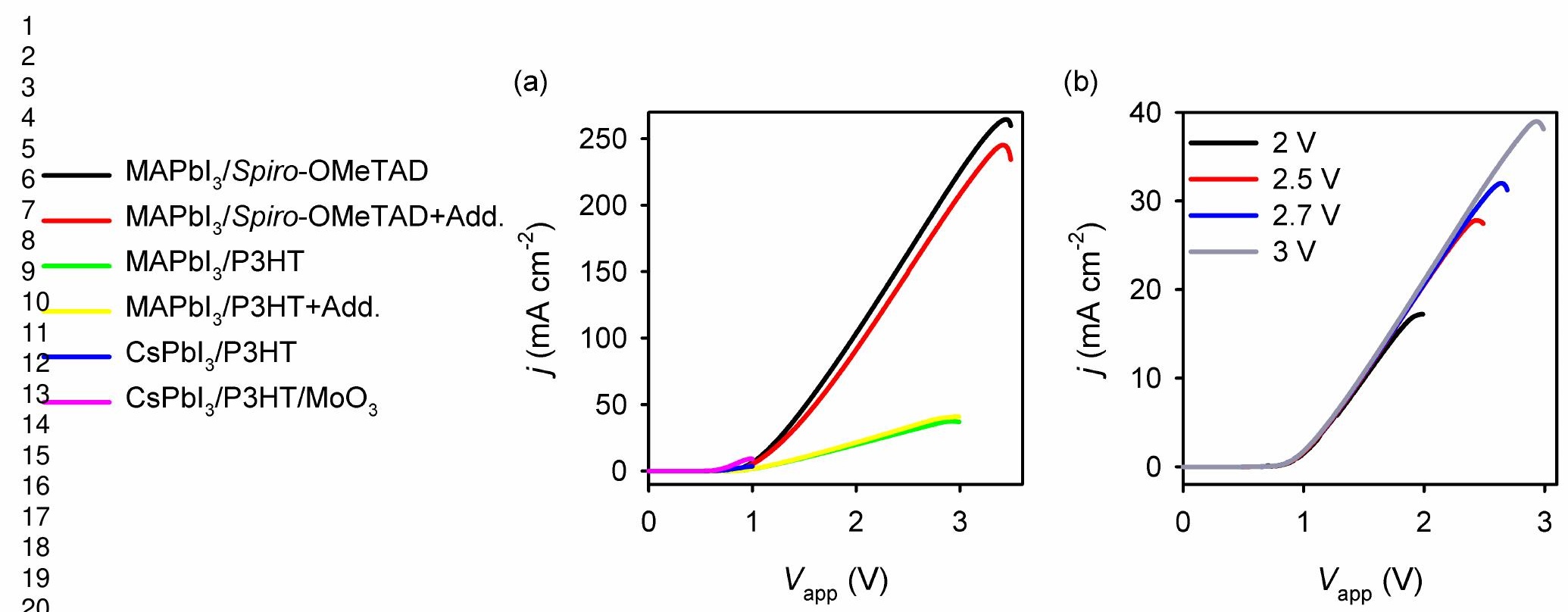

Figure 3 
(a)

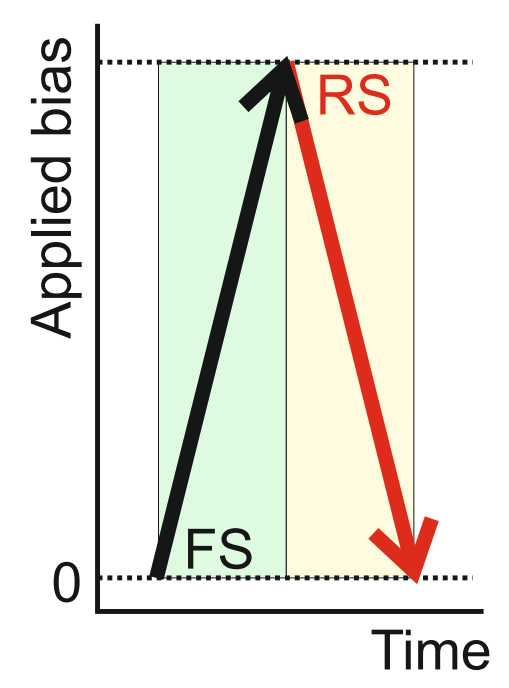

(e)

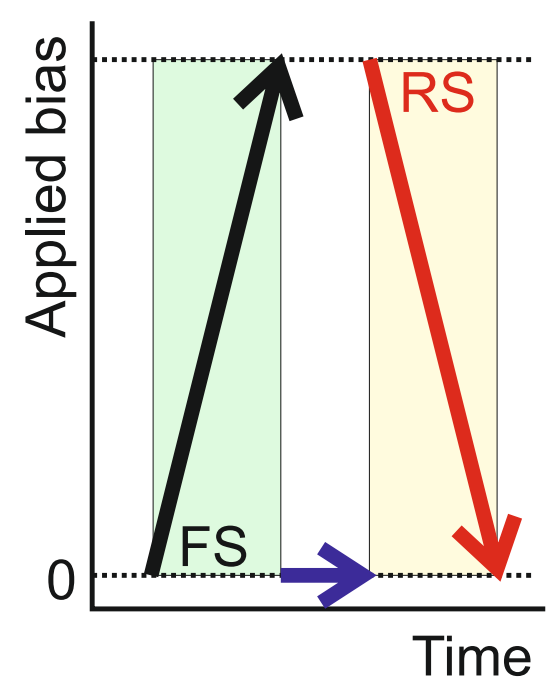

(b)

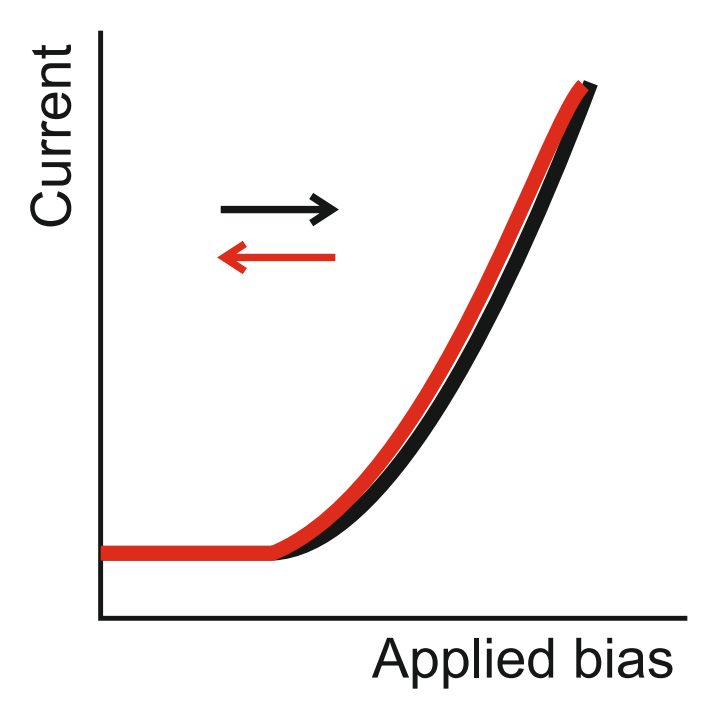

(f)

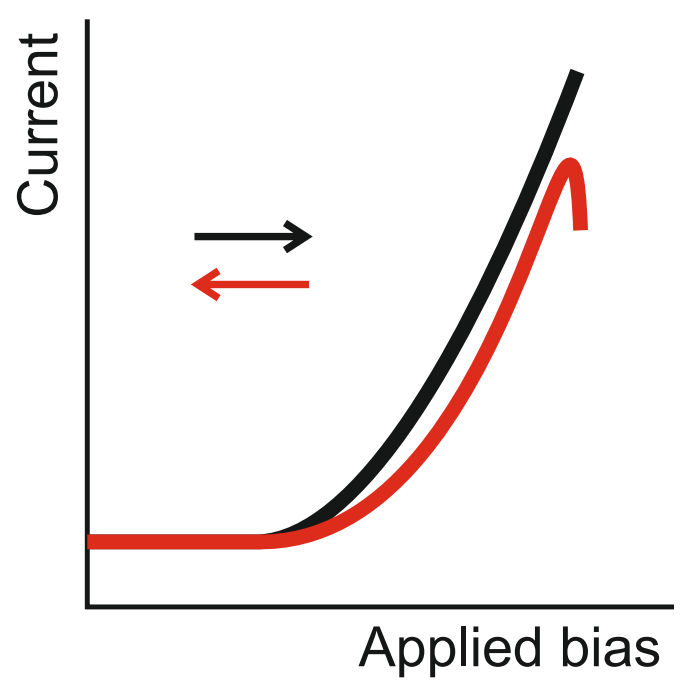

(c)

$V_{\text {app }}$

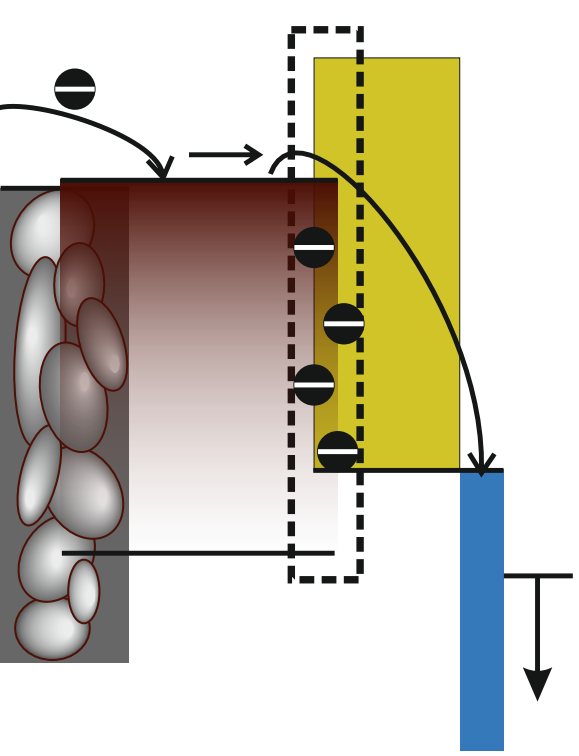

(g)

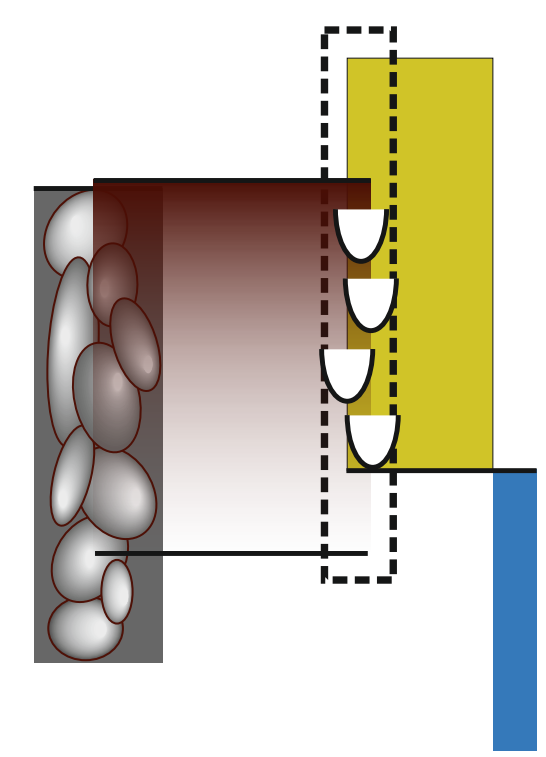

(d)

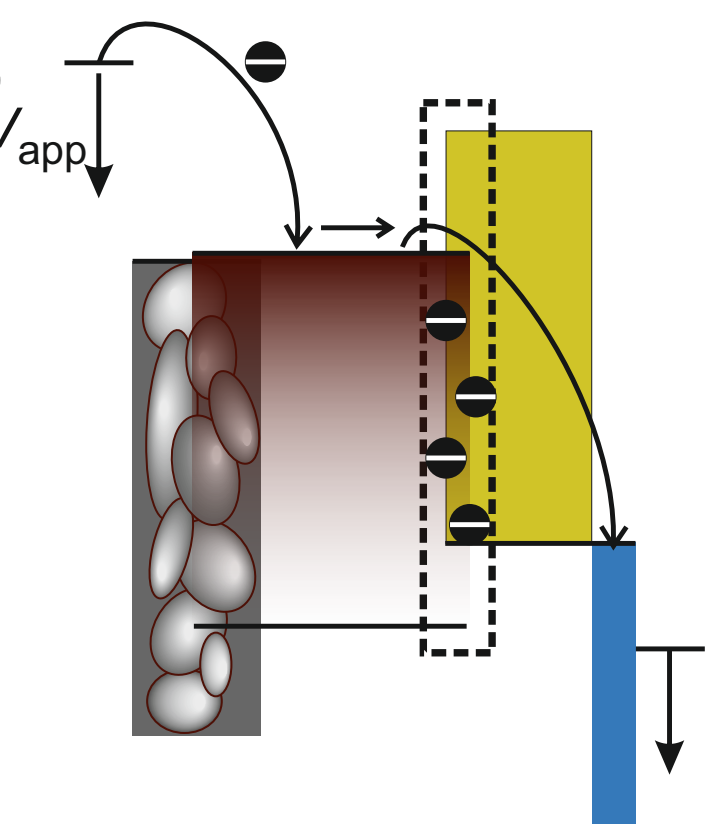

(h)

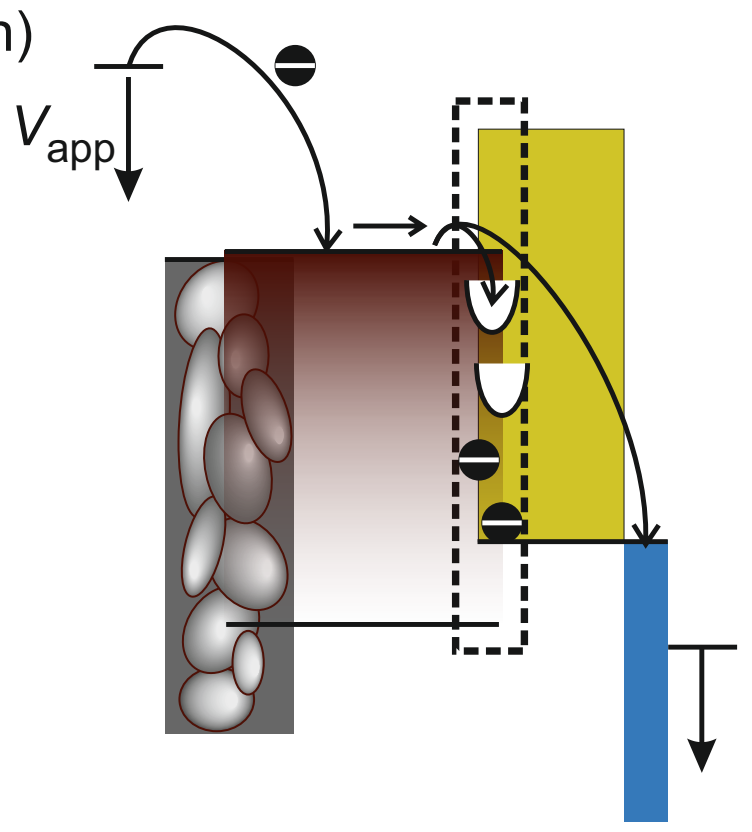

Figure 4 